\title{
CONCERNING CONVERGENCE OF CONTINUED FRACTIONS
}

\author{
DAVID F. DAWSON
}

1. Introduction. For convenience we shall say that the continued fraction

$$
F(c, d)=d_{0}+\frac{c_{0}}{d_{1}}+\frac{c_{1}}{d_{2}}+\frac{c_{2}}{d_{3}}+\cdots
$$

with sequence of approximants $\left\{F_{p}\right\}$ has property (A) means there exists a complex number $v$ such that each of the sequences $\left\{F_{2 p-1}\right\}$ and $\left\{F_{2 p}\right\}$ contains an infinite subsequence convergent to $v$. Here, $c$ denotes the complex number sequence $\left\{c_{p}\right\}_{p=0}^{\infty}$ and $d$ denotes the complex number sequence $\left\{d_{p}\right\}_{p=0}^{\infty}$. When we speak of $F(c, d)$ convergent absolutely at least in the wider sense we shall mean that there exists a positive integer $n$ such that the continued fraction

$$
d_{n-1}+\frac{c_{n-1}}{d_{n}}+\frac{c_{n}}{d_{n+1}}+\frac{c_{n+1}}{d_{n+2}}+\cdots
$$

converges absolutely. It is our purpose in this paper to present the results of a study of these properties and to apply these results to questions of convergence of continued fractions. As a consequence of this investigation we are able to obtain convergence criteria which sharpen several known theorems. Let $f(a)$ denote the continued fraction

$$
\frac{1}{1}+\frac{a_{1}}{1}+\frac{a_{2}}{1}+\frac{a_{3}}{1}+\cdots
$$

One of our results in connection with absolute convergence at least in the wider sense may be stated as follows: If the even (odd) part of $f(a)$ converges absolutely at least in the wider sense, the odd (even) part converges, and $\lim \left|f_{2 p}\right|=\infty\left(\lim \left|f_{2 p-1}\right|=\infty\right)$, then the series $\sum\left|b_{2 p}\right|$ (the series $\left.\sum\left|b_{2 p-1}\right|\right)$ converges and $\lim \sup \left|b_{1}+b_{3}+\cdots+b_{2 p-1}\right|<\infty$

$$
\left(\lim \sup \left|b_{2}+b_{4}+\cdots+b_{2 p}\right|<\infty\right) \text {, }
$$

where $b_{1}=1, b_{p+1}=1 / a_{p} b_{p}, p=1,2,3, \cdots$, and $f_{p}$ is the $p$ th approximant of $f(a)$. This result is used to show that if there exists a sequence $\left\{r_{p}\right\}_{p=1}^{\infty}$ of nonnegative numbers such that

Presented to the Society, April 17, 1959; received by the editors April 7, 1959 and, in revised form, September 11, 1959. 


$$
\begin{aligned}
r_{1}\left|1+a_{1}\right| & \geqq\left|a_{1}\right|, \\
r_{2}\left|1+a_{1}+a_{2}\right| & \geqq\left|a_{2}\right|, \\
r_{p}\left|1+a_{p-1}+a_{p}\right| & \geqq r_{p} r_{p-2}\left|a_{p-1}\right|+\left|a_{p}\right|, p=3,4,5, \cdots,
\end{aligned}
$$

with actual inequality holding either in the first relation or in the second, then either some $a_{p}=0$ and $f(a)$ converges, or else no $a_{p}=0$ and the divergence of the series $\sum\left|b_{p}\right|$ is necessary and sufficient for the convergence of $f(a)$. Lane and Wall [3] required actual inequality in both of the first two relations of (1.1) in case no $a_{p}=0$.

2. Lemmas. Let $g(b)$ denote the continued fraction

$$
\frac{1}{b_{1}}+\frac{1}{b_{2}}+\frac{1}{b_{3}}+\ldots
$$

Our notation in connection with $f(a)$ and $g(b)$ is as follows. If $f_{p}$ and $g_{p}$ are the $p$ th approximants of $f(a)$ and $g(b)$, respectively, then $f_{p}=A_{p} / B_{p}$ and $g_{p}=C_{p} / D_{p}$, where

$$
\begin{aligned}
A_{0}=0, & A_{1}=1, \quad A_{q+1}=A_{q}+a_{q} A_{q-1}, \\
B_{0}=1, & B_{1}=1, \quad B_{q+1}=B_{q}+a_{q} B_{q-1}, \\
C_{0}=0, & C_{1}=1, \quad C_{q+1}=b_{q+1} C_{q}+C_{q-1}, \\
D_{0}=1, & D_{1}=b_{1}, \quad D_{q+1}=b_{q+1} D_{q}+D_{q-1},
\end{aligned}
$$

We note that if $p$ is a positive integer, then

$$
\begin{aligned}
& f_{p+1}-f_{p}=(-1)^{p} \frac{a_{1} a_{2} \cdots a_{p}}{B_{p+1} B_{p}}, \quad \text { if } B_{p}, B_{p+1} \neq 0, \\
& f_{p+2}-f_{p}=(-1)^{p} \frac{a_{1} a_{2} \cdots a_{p}}{B_{p+2} B_{p}}, \quad \text { if } B_{p+2}, B_{p} \neq 0, \\
& g_{p+1}-g_{p}=(-1)^{p} \frac{1}{D_{p+1} D_{p}}, \quad \text { if } D_{p+1}, D_{p} \neq 0 \text {, } \\
& g_{p+1}-g_{p-1}=(-1)^{p+1} \frac{b_{p+1}}{D_{p+1} D_{p-1}}, \quad \text { if } D_{p+1}, D_{p-1} \neq 0 .
\end{aligned}
$$

If $b_{1}=1, a_{p} \neq 0, b_{p+1}=1 / a_{p} b_{p}, p=1,2,3, \cdots$, then $g(b)$ is equivalent to $f(a)$ in that the two continued fractions have the same sequence of approximants. From (2.1) and (2.2) we have:

Lemma 2.1. If the even (odd) part of $f(a)$ converges and some $a_{p}=0$, then $f(a)$ has property (A). 
LEMMA 2.2. If $F(c, d)$ converges absolutely at least in the wider sense and $c_{p} \neq 0, p=0,1,2, \cdots$, then for some positive integer $k$, one of the series $\sum_{(p>k)}\left|F_{p+1}-F_{p}\right|$ and $\sum_{(p>k)}\left|1 / F_{p+1}-1 / F_{p}\right|$ converges, where $1 / F_{i}=0$ if $F_{i}=\infty$.

Proof. The lemma will follow if we can show that if $i$ is a positive integer and the continued fraction

$$
d_{i}+\frac{c_{i}}{d_{i+1}}+\frac{c_{i+1}}{d_{i+2}}+\cdots
$$

or its reciprocal converges absolutely, then the continued fraction

$$
d_{i-1}+\frac{c_{i-1}}{d_{i}}+\frac{c_{i}}{d_{i+1}}+\ldots
$$

or its reciprocal converges absolutely. Obviously, (2.4) converges absolutely in case the reciprocal of (2.3) converges absolutely. Since the reciprocal of a continued fraction converges absolutely in case the continued fraction itself converges absolutely to a value distinct from zero, we need only consider the case that (2.3) converges absolutely to zero. In this case we see that the reciprocal of (2.4) converges absolutely since $c_{i-1} \neq 0$.

3. Results. We shall make use of a formula of Wall [6] which expresses the fact that $a_{p+2}$ is a cross ratio of four successive approximants of $f(a)$. Specifically,

$$
-a_{p+2}\left(f_{p}-f_{p+2}\right)\left(f_{p+1}-f_{p+3}\right)=\left(f_{p}-f_{p+1}\right)\left(f_{p+2}-f_{p+3}\right),
$$

if $B_{p}, B_{p+1}, B_{p+2}$, and $B_{p+3}$ are distinct from zero. This formula follows from (2.2). Our first theorem is a slight extension of a theorem of Wall [6, Theorem 1].

THEOREM 3.1. If the even (odd) part of $f(a)$ converges and there exists $a$ positive integer $k$ such that $B_{p} \neq 0$ for $p>k$, then $f(a)$ has property (A) in case $f(a)$ is partially bounded, i.e., in case lim inf $\left|a_{n}\right|<\infty$.

Proof. If some $a_{p}=0$, then $f(a)$ has property (A) by Lemma 2.1 . Suppose no $a_{p}=0,\left\{f_{2 p}\right\}$ converges to $v$, but $f(a)$ does not have property (A). Then there exists a positive number $R$ and a positive integer $N_{1}$ such that if $p>N_{1}$, then $\left|f_{2 p+1}-v\right|>2 R$. There exists a positive integer $N_{2}$ such that if $p>N_{2}$, then $\left|f_{2 p}-v\right|<R$. Let $N=\max \left[k, N_{1}, N_{2}\right]$. Suppose $p>N$. Then

$$
\begin{aligned}
\left|f_{2 p+1}-v\right| & \leqq\left|f_{2 p+1}-f_{2 p}\right|+\left|f_{2 p}-v\right|<\left|f_{2 p+1}-f_{2 p}\right|+R \\
& <\left|f_{2 p+1}-f_{2 p}\right|+\left|f_{2 p+1}-v\right| / 2
\end{aligned}
$$


and so $\left|f_{2 p+1}-v\right|<2\left|f_{2 p+1}-f_{2 p}\right|$, and similarly

$$
\left|f_{2 p+1}-v\right|<2\left|f_{2 p+1}-f_{2 p+2}\right| \text {. }
$$

Hence by (3.1), if $q>N$, then

$$
\begin{aligned}
\left|a_{2 q+2}\left(f_{2 q}-f_{2 q+2}\right)\left(f_{2 q+1}-f_{2 q+3}\right)\right| & =\left|\left(f_{2 q}-f_{2 q+1}\right)\left(f_{2 q+2}-f_{2 q+3}\right)\right| \\
& >\left|f_{2 q+1}-v\right|\left|f_{2 q+3}-v\right| / 4,
\end{aligned}
$$

and so

$$
\begin{aligned}
& \left|f_{2 q+1}-v\right|\left|f_{2 q+3}-v\right| \\
& \quad<4\left|a_{2 q+2}\right|\left|f_{2 q}-f_{2 q+2}\right|\left[\left|f_{2 q+1}-v\right|+\left|f_{2 q+3}-v\right|\right] .
\end{aligned}
$$

If we divide both sides of this inequality by the larger of $\left|f_{2 q+1}-v\right|$ and $\left|f_{2 q+3}-v\right|$, we find that $R<4\left|a_{2 q+2}\right|\left|f_{2 q}-f_{2 q+2}\right|$. Similarly $R<4\left|a_{2 q+3}\right|\left|f_{2 q+2}-f_{2 q+4}\right|$. But this is impossible if $f(a)$ is partially bounded and $\left\{f_{2 p}\right\}$ converges. Hence the theorem is established for the case that we have considered. A similar argument can be used in case $\left\{f_{2 p-1}\right\}$ converges.

As an application of Theorem 3.1, we consider the following. Suppose the inequalities (1.1) hold. It has been shown [4] that $B_{p} \neq 0$, $p=0,1,2, \cdots$. Thus if some $a_{p}=0$, then $f(a)$ converges $[5$, p. 26]. Suppose no $a_{p}=0$. It is known $[4 ; 3]$ that $\left\{f_{2 p-1}\right\}$ and $\left\{f_{2 p}\right\}$ converge at least in the wider sense and if $r_{1}\left|1+a_{1}\right|>\left|a_{1}\right|$, then $\left\{f_{2 p}\right\}$ converges, and if $r_{2}\left|1+a_{1}+a_{2}\right|>\left|a_{2}\right|$, then $\left\{f_{2 p-1}\right\}$ converges. We shall suppose here that actual inequality holds either in the first relation of (1.1) or in the second. If we impose the condition that $f(a)$ be partially bounded, then we see that the conditions of Theorem 3.1 are satisfied with one of the sequences $\left\{f_{2 p-1}\right\}$ and $\left\{f_{2 p}\right\}$ convergent and the other convergent at least in the wider sense. But $f(a)$ has property (A) and consequently must converge. Hence we have:

Corollary 3.1a. If the inequalities (1.1) hold with actual inequality in the first relation or in the second, then $f(a)$ converges if $f(a)$ is partially bounded.

This result extends or includes the following theorems of [4]: Theorem 3.3, Theorem 3.4, and Theorem E.

Corollary 3.1b. If the even (odd) part of $f(a)$ converges and the sequence $\left\{a_{p}\right\}$ is bounded, then $f(a)$ has property (A).

Proof. Suppose the even part of $f(a)$ converges. If there exists a positive integer $k$ such that $B_{p} \neq 0$ for $p>k$, then $f(a)$ has property (A) by Theorem 3.1. Suppose no such $k$ exists. Let $M$ be a number such that $\left|a_{p}\right|<M, p=1,2,3, \cdots$. Let $i$ be an odd positive integer such that $B_{i}=0$. Then by (2.1), 


$$
\begin{aligned}
& B_{i+1}=B_{i}+a_{i} B_{i-1}=a_{i} B_{i-1}, \\
& B_{i+2}=B_{i+1}+a_{i+1} B_{i}=B_{i+1},
\end{aligned}
$$

and we see that $a_{i} \neq 0, B_{i-1} \neq 0$, and $B_{i+1} \neq 0$, for if any one of these quantities were zero, then we would have two consecutive $B$ 's equal to zero and the even part of $f(a)$ could not converge. Hence by (2.1),

$$
\begin{aligned}
A_{i+2} & =A_{i+1}+a_{i+1} A_{i}, \\
A_{i+2} / B_{i+2} & =A_{i+1} / B_{i+1}+a_{i+1} A_{i} / B_{i+1}, \\
A_{i+1} & =A_{i}+a_{i} A_{i-1}, \\
A_{i+1} / B_{i+1} & =A_{i} / B_{i+1}+A_{i-1} / B_{i-1},
\end{aligned}
$$

and so

$$
\left|A_{i+2} / B_{i+2}-A_{i+1} / B_{i+1}\right|<M\left|A_{i+1} / B_{i+1}-A_{i-1} / B_{i-1}\right| .
$$

Since this inequality holds for infinitely many values of $i$, we see that $f(a)$ has property (A). A similar proof can be used if we assume that the odd part of $f(a)$ converges. Thus the corollary is established.

THEOREM 3.2. If the even (odd) part of $f(a)$ converges absolutely at least in the wider sense, the odd (even) part converges, and $\lim \left|f_{2 p}\right|$ $=\infty\left(\lim \left|f_{2 p-1}\right|=\infty\right)$, then the series $\sum\left|b_{2 p}\right|$ (the series $\left.\sum\left|b_{2 p-1}\right|\right)$ converges and

$\lim \sup \left|b_{1}+b_{3}+\cdots+b_{2 p-1}\right|<\infty$

$$
\left(\lim \sup \left|b_{2}+b_{4}+\cdots+b_{2 p}\right|<\infty\right) \text {, }
$$

where $b_{1}=1, b_{p+1}=1 / a_{p} b_{p}, p=1,2,3, \cdots$.

PROOF. If some $a_{p}=0$, then $f(a)$ has property (A) by Lemma 2.1, contrary to hypothesis. We shall prove the theorem for the case that the even part of $f(a)$ converges absolutely at least in the wider sense, etc. The other case is similar. We shall consider the continued fraction $g(b)$ which is equivalent to $f(a)$. We observe that since $\left|C_{p+1} D_{p}-C_{p} D_{p+1}\right|=1$ for all $p$ and $\lim \left|f_{2 p}\right|=\infty$, then there exists a positive integer $N_{1}$ such that if $p \geqq N_{1}$, then $C_{2 p} \neq 0$. If $p>N_{1}$, then

$$
\begin{aligned}
\left|1 / f_{2 p+2}-1 / f_{2 p}\right| & =\left|D_{2 p+2} / C_{2 p+2}-D_{2 p} / C_{2 p}\right| \\
& =\left|b_{2 p+2}\right| /\left|C_{2 p+2} C_{2 p}\right| .
\end{aligned}
$$

There exists a positive integer $N_{2}$ and a positive number $k$ such that if $p>N_{2}$, then $D_{2 p+1} \neq 0$ and either $C_{2 p+1}=0$ or $\left|1 / f_{2 p+2}-1 / f_{2 p+1}\right|>k$ and $\left|1 / f_{2 p}-1 / f_{2 p+1}\right|>k$. Let $N=\max \left[N_{1}, N_{2}\right]$. Suppose $p>N$. If $C_{2 p+1} \neq 0$, then

$$
\begin{aligned}
k<\left|1 / f_{2 p+2}-1 / f_{2 p+1}\right| & =\left|D_{2 p+2} / C_{2 p+2}-D_{2 p+1} / C_{2 p+1}\right| \\
& =1 /\left|C_{2 p+2} C_{2 p+1}\right|
\end{aligned}
$$


and so $\left|C_{2 p+2} C_{2 p+1}\right|<1 / k$. Thus

$$
\begin{aligned}
\left|1-C_{2 p+2} / C_{2 p}\right| & =\left|b_{2 p+2} C_{2 p+1} C_{2 p+2} / C_{2 p} C_{2 p+2}\right| \\
& =\left|1 / f_{2 p+2}-1 / f_{2 p}\right|\left|C_{2 p+1} C_{2 p+2}\right| \\
& <(1 / k)\left|1 / f_{2 p+2}-1 / f_{2 p}\right| .
\end{aligned}
$$

By Lemma 2.2 the series $\sum_{(p>N)}\left|1 / f_{2 p+2}-1 / f_{2 p}\right|$ converges and so the series $\sum_{(p>N)}\left|1-C_{2 p+2} / C_{2 p}\right|$ converges, since if $p>N$ and $C_{2 p+1}=0$, then $\left|1-C_{2 p+2} / C_{2 p}\right|=0$. Thus by Lemma 2.1 of [1], the series $\sum\left|C_{2 p}-C_{2 p+2}\right|$ converges. Therefore by (3.2) there exists a positive integer $t$ and a positive number $M$ such that if $p>t$, then $\left|b_{2 p+2}\right| \leqq M\left|1 / f_{2 p+2}-1 / f_{2 p}\right|$. Thus the series $\sum\left|b_{2 p}\right|$ converges. Suppose $p>N$ and $C_{2 p+1} \neq 0$. Then from (3.2),

$$
\begin{aligned}
\left|1 / f_{2 p+2}-1 / f_{2 p}\right| & =\left|b_{2 p+2}\right| /\left|C_{2 p+2} C_{2 p}\right| \\
& =\left|b_{2 p+2}\right|\left|C_{2 p+1}\right|^{2} /\left|C_{2 p+2} C_{2 p+1}\right|\left|C_{2 p+1} C_{2 p}\right|,
\end{aligned}
$$

and from (3.3),

$$
\left|1 / f_{2 p+2}-1 / f_{2 p}\right| \geqq k^{2}\left|b_{2 p+2}\right|\left|C_{2 p+1}\right|^{2} .
$$

Thus the series $\sum\left|b_{2 p+2}\right|\left|C_{2 p+1}\right|^{2}$ converges. Using summation by parts we note that if $n>N$, then

$$
\begin{aligned}
\sum_{p=N}^{n}\left[C_{2 p+1}-C_{2 p-1}\right]\left(1 / C_{2 p}\right)= & C_{2 n+1} / C_{2 n}-C_{2 N-1} / C_{2 N} \\
& +\sum_{p=N}^{n-1} C_{2 p+1}\left[1 / C_{2 p}-1 / C_{2 p+2}\right],
\end{aligned}
$$

and so

$$
\sum_{p=N}^{n} b_{2 p+1}=C_{2 n+1} / C_{2 n}-C_{2 N-1} / C_{2 N}+\sum_{p=N}^{n-1} b_{2 p+2} C_{2 p+1}^{2} / C_{2 p} C_{2 p+2} .
$$

From (3.3) and the fact that $\left\{C_{2 p}\right\}$ converges to a point not zero [2], we see that $\lim \sup \left|C_{2 p+1}\right|<\infty$. Thus $\lim$ sup $\left|b_{1}+\cdots+b_{2 p-1}\right|$ $<\infty$, and the theorem is proved.

Since, in the proof of Theorem 3.2, after disposing of the case that some $a_{p}=0$, we considered the continued fraction $g(b)$ equivalent to $f(a)$ and made no use of the fact that no $b_{p}=0$, we have:

COROLlaRY 3.2a. If $\left\{g_{2 p}\right\}\left(\left\{g_{2 p-1}\right\}\right)$ converges absolutely at least in the wider sense, $\left\{g_{2 p-1}\right\} \quad\left(\left\{g_{2 p}\right\}\right)$ converges, and

$$
\lim \left|g_{2 p}\right|=\infty\left(\lim \left|g_{2 p-1}\right|=\infty\right),
$$

then the series $\sum\left|b_{2 p}\right|$ (the series $\left.\sum\left|b_{2 p-1}\right|\right)$ converges and $\lim \sup \left|b_{1}+\cdots+b_{2 p-1}\right|<\infty\left(\lim \sup \left|b_{2}+\cdots+b_{2 p}\right|<\infty\right)$. 
THEOREM 3.3. If the even (odd) part of $f(a)$ converges absolutely, the odd (even) part converges absolutely at least in the wider sense, and $\lim \left|f_{2 p-1}\right|=\infty\left(\lim \left|f_{2 p}\right|=\infty\right)$, then the series $\sum\left|b_{p}\right|$ converges, where $b_{1}=1, b_{p+1}=1 / a_{p} b_{p}, p=1,2,3, \cdots$.

Proof. If some $a_{p}=0$, then $f(a)$ has property (A) by Lemma 2.1, contrary to hypothesis. We shall prove the theorem for the case that the even part converges absolutely, the odd part converges absolutely at least in the wider sense, etc. The other case is similar. In [1] we showed that if the even (odd) part of $f(a)$ converges absolutely and the series $\sum\left|b_{2 p}\right|$ (the series $\sum\left|b_{2 p-1}\right|$ ) diverges, then $f(a)$ has property (A). Thus for the case we are considering, the series $\sum\left|b_{2 p}\right|$ converges. The fact that the series $\sum\left|b_{2 p-1}\right|$ converges follows from Theorem 3.2. Thus the theorem is established.

As an extension of Theorem 2.4 of [3] we have:

CoROllary 3.3a. If the even (odd) part of $f(a)$ converges absolutely and the odd (even) part converges absolutely at least in the wider sense, then either some $a_{p}=0$ and $f(a)$ converges, or else no $a_{p}=0$ and the divergence of the series $\sum\left|b_{p}\right|$ is necessary and sufficient for the convergence of $f(a)$.

As an application of this result we have:

CoRollary 3.3b. If the inequalities (1.1) hold with actual inequality holding either in the first relation or in the second, then either some $a_{p}=0$ and $f(a)$ converges, or else no $a_{p}=0$ and the divergence of the series $\sum\left|b_{p}\right|$ is necessary and sufficient for the convergence of $f(a)$.

Proof. Apply Corollary 3.3a after noting that one of the sequences $\left\{f_{2 p}\right\}$ and $\left\{f_{2 p-1}\right\}$ converges absolutely and the other converges absolutely at least in the wider sense.

This result extends Theorem 4.1 of [3] and our Corollary 3.1a.

We next consider a theorem concerning absolute convergence of $f(a)$. The basic idea is due to Lane and Wall [3].

THEOREM 3.4. If there exist numbers $k$ and $M$ and a sequence $\left\{p_{i}\right\}_{i=1}^{\infty}$ of positive integers such that for each positive integer $i, 0<p_{i+1}$ $-p_{i}<k$ and $\left|a_{p_{i}}\right|<M$, then $f(a)$ converges absolutely if the even and odd parts of $f(a)$ converge absolutely.

Proof. For simplicity assume that $p_{1}>2$. Let $N$ be a positive integer such that if $p>N$, then $B_{p-2} \neq 0$. From (3.1), if $i>N$, then

$$
\left|f_{p_{i}-2}-f_{p_{i}-1}\right|\left|f_{p_{i}}-f_{p_{i}+1}\right|=\left|a_{p_{i}}\right|\left|f_{p_{i}-2}-f_{p_{i}}\right|\left|f_{p_{i}-1}-f_{p_{i}+1}\right| \text {, }
$$

and so, 


$$
\begin{aligned}
\min \left[\left|f_{p_{i}-2}-f_{p_{i}-1}\right|,\right. & \left.\left|f_{p_{i}}-f_{p_{i}+1}\right|\right] \\
& \leqq M^{1 / 2} \max \left[\left|f_{p_{i}-2}-f_{p_{i}}\right|,\left|f_{p_{i}-1}-f_{p_{i}+1}\right|\right] .
\end{aligned}
$$

Let $\left|f_{q_{i}}-f_{q_{i}+1}\right|$ denote $\min \left[\left|f_{p_{i}-2}-f_{p_{i}-1}\right|,\left|f_{p_{i}}-f_{p_{i}+1}\right|\right]$. Then either $q_{i}=p_{i}-2$ or $q_{i}=p_{i}$. Also let $S_{p_{i}}$ denote

$$
\left(1+M^{1 / 2}\right)\left[\left|f_{p_{i}-1}-f_{p_{i}+1}\right|+\cdots+\left|f_{p_{i+1}-1}-f_{p_{i+1}+1}\right|\right] .
$$

Clearly the series $\sum S_{p_{i}}$ converges. If $p_{i}-1 \leqq j \leqq p_{i+1}-2$, then

$$
\begin{aligned}
\left|f_{j}-f_{j+1}\right| \leqq & \left|f_{j}-f_{j+2}\right|+\left|f_{j+1}-f_{j+3}\right| \\
& +\cdots+\left|f_{q_{i+1}-1}-f_{q_{i+1}+1}\right|+\left|f_{q_{i+1}}-f_{q_{i+1}+1}\right| \\
\leqq & \left|f_{j}-f_{j+2}\right|+\cdots+\left|f_{q_{i+1}-1}-f_{q_{i+1}+1}\right| \\
& +M^{1 / 2}\left[\left|f_{p_{i+1}-2}-f_{p_{i+1}}\right|+\left|f_{p_{i+1}-1}-f_{p_{i+1}+1}\right|\right] \\
\leqq & S_{p_{i} .}
\end{aligned}
$$

Hence

$$
\left|f_{p_{i}-1}-f_{p_{i}}\right|+\left|f_{p_{i}}-f_{p_{i}+1}\right|+\cdots+\left|f_{p_{i+1}-2}-f_{p_{i+1}-1}\right| \leqq k S_{p_{i}} .
$$

Thus we see that $f(a)$ converges absolutely.

As an extension of Theorem 6.1 of [3] we have:

CoRollary 3.4a. If there exist numbers $k$ and $M$ such that $\left|a_{p}\right|<M$ for the values of $p$ specified in Theorem 3.4, and if the inequalities (1.1) hold with actual inequality in the first relation or in the second, then $f(a)$ converges absolutely.

Proof. Follows directly from Theorems 3.1 and 3.4.

\section{REFERENCES}

1. D. F. Dawson, Continued fractions with absolutely convergent even or odd part, Canad. J. Math. vol. 11 (1959) pp. 131-140.

2. - Convergence of continued fractions of Stieltjes type, Proc. Amer. Math. Soc. vol. 10 (1959) pp. 12-17.

3. R. E. Lane and H. S. Wall, Continued fractions with absolutely convergent even and odd parts, Trans. Amer. Math. Soc. vol. 67 (1949) pp. 368-380.

4. W. T. Scott and H. S. Wall, A convergence theorem for continued fractions, Trans. Amer. Math. Soc. vol. 47 (1940) pp. 155-172.

5. H. S. Wall, Analytic theory of continued fractions, New York, 1948.

6. - Partially bounded continued fractions, Proc. Amer. Math. Soc. vol. 7 (1956) pp. 1090-1093.

\section{UNIVERSITY OF MISSOURI AND}

North Texas State College 